\title{
The Demand Side Management Potential to Balance a Highly Renewable European Power System
}

\author{
Alexander Kies ${ }^{1,2, *}$, Bruno U. Schyska ${ }^{1,2}$ and Lueder von Bremen ${ }^{1}$ \\ 1 ForWind, Center for Wind Energy Research, Küpkersweg 70, 26129 Oldenburg, Germany; \\ bruno.schyska@forwind.de (B.U.S.); lueder.von.bremen@forwind.de (L.v.B.) \\ 2 Institute of Physics, University of Oldenburg, Ammerländer Heerstr. 114, 26129 Oldenburg, Germany \\ * Correspondence: alexander.kies@uni-oldenburg.de
}

Academic Editor: William Holderbaum

Received: 03 July 2016; Accepted: 08 November 2016; Published: 15 November 2016

\begin{abstract}
Shares of renewables continue to grow in the European power system. A fully renewable European power system will primarily depend on the renewable power sources of wind and photovoltaics (PV), which are not dispatchable but intermittent and therefore pose a challenge to the balancing of the power system. To overcome this issue, several solutions have been proposed and investigated in the past, including storage, backup power, reinforcement of the transmission grid, and demand side management (DSM). In this paper, we investigate the potential of DSM to balance a simplified, fully renewable European power system. For this purpose, we use ten years of weather and historical load data, a power-flow model and the implementation of demand side management as a storage equivalent, to investigate the impact of DSM on the need for backup energy. We show that DSM has the potential to reduce the need for backup energy in Europe by up to one third and can cover the need for backup up to a renewable share of $67 \%$. Finally, it is demonstrated that the optimal mix of wind and PV is shifted by the utilisation of DSM towards a higher share of PV, from $19 \%$ to $36 \%$.
\end{abstract}

Keywords: demand side management; renewable energy systems; European power system; energy system modelling; wind energy; solar energy

\section{Introduction}

Aiming at sustainability and reduced $\mathrm{CO}_{2}$ emissions, shares of renewable generation are on the rise all across Europe. This is in line with the 2015 United Nations Climate Change Conference (CMP 11) commitments. However, the integration of intermittent renewable generation from wind and photovoltaics (PV) into energy systems poses severe balancing challenges [1]. Unlike conventional generation (e.g., nuclear, fossil, etc.), renewable generation is driven by the weather and can not be reliably dispatched and therefore not directly adopted to follow the demand. Several possible approaches to this issue have been investigated: (i) Optimising the mix of different renewable sources [2-8]; (ii) Storage to shift generation in time [9,10]; (iii) Backup [11-13]; (iv) Or the reinforcement of the transmission grid to shift generation in space [14,15]. In addition to these approaches on the generation side, part of the need for balancing might also be covered by the modification of the demand for power on the consumer side with the objective of increasing its manageability (e.g., to match renewable generation in time $[16,17])$. One definition of demand side management (DSM) is "the planning, implementation, and monitoring of utility activities designed to encourage consumers to modify patterns of electricity usage, including the timing and level of energy demand." [18]. The interest in different aspects of DSM has risen in recent years, along with the rising general interest in renewable power systems. DSM storage strategies for end-users were investigated in [19]. Large-scale industrial processes might be able to provide up to $50 \%$ of backup capacity need 
by 2020 [20,21]. Furthermore, DSM can significantly reduce the need for conventional generation [22] even on a residential scale. It is expected that most contributions to a flexible demand side will be provided by industry. In Germany, for instance, a major contributor might be the automotive industry [23]. Klobasa et al. [24] investigate the interplay of load management and wind power forecasts in Germany and concludes that load management can reduce balancing costs by up to $20 \%$ and is mostly economically useful. Lund et al. [25] investigate the potential impact of electric cars in a vehicle-to-grid (V2G) system on a renewable Danish power system. Stadler et al. [26] conclude that different DSM branches can complement each other fairly well in Germany to provide seasonally independent power and that the biggest fraction of demand side management can be provided by storage heating and combined heat and power (CHP). Moura et al. [27] use a heuristic approach to show a reduction of Portuguse peak loads of more than $10 \%$ through the use of DSM. A similar heuristic approach to characterise the potential of DSM is applied in [28]. A broad overview of different DSM types is given in [29]. In [30], an energy system model is used to estimate the impact of DSM in the EU-27. However, it only inhibits a low renewable share in the system and focuses on congestion. This model is methodologically extended in [31].

There are many studies that investigate aspects of the potential of demand side management in Europe, and most of them conclude that DSM can contribute significantly to a reliable renewable energy supply, but they only provide rough estimates of the benefit [32,33]. However, we are not aware of any studies that show the overall impact of DSM on the need for backup energy by a large scale integration into a fully renewable European power system in a systematic way as we did in this paper.

In this paper, the novel framework described in [34] is used to implement DSM into a model of a fully renewable European power system with country-level resolution. All of the DSM potentials of a single country are treated as one large storage-equivalent with time dependent constraints. Weather data is used to model feed-in from the renewable sources of wind and PV. Together with historical load data and a power flow model, the need for backup energy was calculated and the impact of DSM was investigated.

We do not include hydropower into the simulations, although it already contributes approximately $10 \%$ to the European electricity mix today. This is because European hydropower, with its seasonal storage characteristics, would likely be used after DSM with its daily storage characteristics, and, therefore, has little effect on the results, but instead replaces a large share of the need for backup energy.

This paper focuses on the following objectives: (i) What is the potential of DSM to reduce the need for backup energy in a fully renewable European power system? (ii) Until which amount of renewable generation can DSM replace backup? and (iii) How does the successful integration of DSM affect the optimal mix of wind and PV generation?

This paper is structured in the following way: first, the model is described in detail. This includes a description of the main components renewable generation and load, the transmission model, and the incorporation of demand side management. Second, the potential benefit of DSM for two scenarios of generation capacity distribution and two scenarios of transmission grid strength is investigated. Third, the impact of the full utilisation of DSM on the optimal share of wind and PV is studied. For this, all generation, load, and DSM potentials are aggregated into a single European node (copper plate approximation). Finally, the potential reduction of the need for backup energy in dependency of the renewable share is investigated.

\section{Model Description}

A highly renewable European power system covering 33 countries was simulated. Every country was aggregated into a single node, and the countries are interconnected via transmission links (Figure 1). Every node $n$ has a generation time series $G_{n}(t)$ from the renewable sources of wind and PV, derived from weather data, and a load time series $L_{n}(t)$, which consists of historical data. 
We used ten years of data ranging from 2003-2012 for all of the following computations. The time series of the mismatch between generation and load of country $n$ is given by

$$
\Delta_{n}(t)=G_{n}(t)-L_{n}(t)
$$

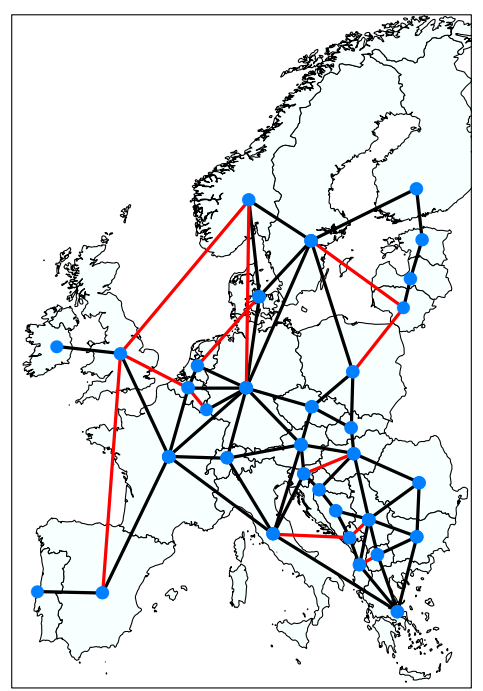

Figure 1. Topology of the investigated simplified European power system. Countries are modeled as nodes and connected by inter-country transmission links. Black links are existing connections, and red ones are either planned or under construction.

At each node and at all times, the power system must be balanced. This is expressed in the nodal balancing equation

$$
G_{n}(t)-L_{n}(t)=\Phi_{n}(t)-B_{n}(t)+C_{n}(t)+S_{n}(t)
$$

$B_{n}(t)$ is the time series of backup, $C_{n}(t)$ is the excess energy that is curtailed and $\Phi_{n}(t)$ is the injection pattern (Exports-Imports). $S_{n}(t)$ is the interaction (charge/discharge) with the storage-equivalent DSM. After transmission and DSM, the remaining residual mismatch is handled by backup, which is assumed to be perfectly flexible, i.e., neither subject to ramping nor must-run constraints. Thus, the backup time series is calculated as

$$
B_{n}(t)=\max \left(\left\{0, L_{n}(t)-G_{n}(t)+\Phi_{n}(t)+S_{n}(t)\right\}\right)
$$

Consequently, the backup energy need in a given period of time $T$ is given by

$$
B_{n}^{E}=\int_{T} B_{n}(t) d t
$$

In reality, backup energy could, for example, be provided by dispatchable gas power plants. The time series for curtailment is given by

$$
C_{n}(t)=\max \left(\left\{0, G_{n}(t)-L_{n}(t)-\Phi_{n}(t)-S_{n}(t)\right\}\right)
$$

Hence, either backup (if $\Delta_{n}(t)<0$ ) or curtailment $\left(\Delta_{n}(t)>0\right)$ occurs at a node $n$. For example, curtailment can be realised by feathering wind turbine blades. The share of renewable generation of a node is denoted as $\alpha_{n}$ and is defined via 


$$
\alpha_{n}=\frac{\left\langle G_{n}(t)\right\rangle}{\left\langle L_{n}(t)\right\rangle}
$$

Equivalently, the share of renewable generation of the whole system consisting of multiple nodes is given by

$$
\alpha=\sum_{n} \alpha_{n} \frac{\left\langle L_{n}\right\rangle}{\langle L\rangle}
$$

Throughout this paper, the terminology "dispatchable generation" refers to power from sources that could be dispatched (e.g., gas). "Backup" refers to the fraction of the dispatchable generation that is needed to cover the intermittency of the renewable generation. For example, if $\alpha=0.7$, we have an average renewable share of $70 \%$ and at least $30 \%$ from dispatchable sources.. The part of dispatchable generation that is needed in addition is referred to as "backup" or "backup energy".

\subsection{Generation and Load Data}

Feed-in from the renewable sources of wind and PV was simulated using a ten-year weather database with a spatial resolution of $7 \times 7 \mathrm{~km}$ and an hourly temporal resolution. Wind speed (and $2 \mathrm{~m}$ temperature) was downscaled from MERRA reanalysis [35] and converted to wind power through the use of an Enercon E-126 power curve with 5\% plain losses. Surface irradiance was calculated using the Heliosat method [36,37] from satellite pictures (Meteosat First Generation, Meteosat Second Generation). To obtain irradiation on the tilted modules, the Klucher model was applied [38]. Detailed information on the database is given in [39]. Finally, generation was aggregated from the $7 \times 7 \mathrm{~km}$ grid to the country level.

For load time series of all considered European countries, historical data provided by the European Network of Transmission System Operators for Electricity (ENTSO-E) was used. This data was split into different load categories and modified to account for expected future changes caused by the increased use of heat pumps and e-mobility within the RESTORE 2050 project.

\subsection{Demand Side Management}

To incorporate demand side management into our model, the methodology of [34] is adopted and described in this section. Equations (8)-(15) are taken from [34]. In addition, a simple example is given in that paper. In this methodology, DSM is treated like storage with time dependent charging and energy constraints. The load time series can be split into different categories, i.e., it is composed of load time series of different categories $L_{n}^{c}(t), L_{n}(t)=\sum_{c} L_{n}^{c}(t)+L_{n}^{\text {stat. }}(t)$. This load is referred to in the following as scheduled load. $L_{n}^{\text {stat. }}$ refers to the part of the load that can not be shifted. It implicitly contains the DSM utilisation shares of different DSM categories, which are given in Table 1. Without DSM, each country would simply have one (scheduled) load time series $L_{n}(t)$.

DSM allows for replacing a scheduled load $L_{n}^{c}(t)$ by a realized load $R_{n}^{c}(t)$. The difference is the charging or discharging rate of the storage-equivalent DSM buffer

$$
P_{n}^{c}\left[R_{n}^{c}(t)\right](t)=R_{n}^{c}(t)-L_{n}^{c}(t)
$$

The square brackets indicate that it takes a function, i.e., the realized load, as an argument. Because we assume no impact of DSM usage on overall energy consumption, a DSM storage filling level can be calculated as the temporal integral over the charging rate

$$
E_{n}^{c}\left[R_{n}^{c}(t)\right](t)=\int_{0}^{t} P_{n}^{c}\left[R_{n}^{c}\left(t^{\prime}\right)\right]\left(t^{\prime}\right) d t^{\prime}
$$


This storage-equivalent differs from a classical storage (e.g., a battery system or pumped hydro plant) by the time dependency of its filling level and charging limits. These time dependent constraints of charging and filling level of the DSM buffer are defined as

$$
\begin{aligned}
& E_{n}^{c,+}(t)=\int_{t}^{t+\Delta t^{c}} L_{n}^{c}\left(t^{\prime}\right) d t^{\prime} \\
& E_{n}^{c,-}(t)=-\int_{t-\Delta t^{c}}^{t} L_{n}^{c}\left(t^{\prime}\right) d t^{\prime} \\
& P_{n}^{c,+}(t)=\Lambda_{n}^{c}(t)-L_{n}^{c}(t) \\
& P_{n}^{c,-}(t)=-L_{n}^{c}(t)
\end{aligned}
$$

Upper limits are indicated by the index ${ }^{+}$and lower limits indicated by the index ${ }^{-} . \Delta t^{c}$ is the time frame of management of a category and $\Lambda_{n}^{c}$ the maximal realisable load. All realised loads within the imposed constraints are valid:

$$
\begin{aligned}
& E_{n}^{c,+}(t) \geq E\left[R_{n}^{c}(t)\right](t) \geq E_{n}^{c,-}(t) \\
& P_{n}^{c,+}(t) \geq P\left[R_{n}^{c}(t)\right](t) \geq P_{n}^{c,-}(t)
\end{aligned}
$$

Time series of the DSM constraints of different categories for the different countries were developed within RESTORE 2050 and are described in [40]. Five categories were defined with individual time frames of management and utilisation shares (Table 1). Utilisation shares determine what share of a category is available for DSM.

Table 1. Load categories defined for demand side management [40].

\begin{tabular}{ccc}
\hline Category & $\boldsymbol{\Delta} \boldsymbol{t}^{\boldsymbol{c}} \mathbf{( h )}$ & Utilisation (\%) \\
\hline Industrial bandload & 4 & 25 \\
Cooling & 1 & 12 \\
Households & 12 & 10 \\
Heat pumps & 24 & 100 \\
E mobility & 6 & 80 \\
\hline
\end{tabular}

How are the charging rates and thus the usage of DSM determined in our model? Since rescheduling of loads is assumed to leave the total energy demand unchanged, DSM does neither cause losses or gains. Hence, the simple assumption is made that local excess energy after transmission is used to charge and local deficits used to discharge the DSM buffer within the constraints. However, in a real-world power market, backup and curtailment might be a more convenient option than DSM. Then again, this study aims at the theoretical potential of DSM. Whether it can be fully exploited, depends strongly on the market conditions.

The algorithm to distribute the charging/discharging among categories consists of the following five steps for time $t$ and node $n$ :

(i) Compute the ratio of the power limit to remaining energy storage for each category

$$
\text { ratio }=\left\{\begin{array}{l}
\frac{P_{n}^{c,-}(t)}{E_{n}^{c}(t)-E_{n}^{c,-}}, \text { if }\left(G_{n}(t)-L_{n}(t)-\Phi_{n}(t)\right)<0 \\
\frac{P_{n}^{c,+}(t)}{E_{n}^{c,+}-E_{n}^{c}(t)}, \text { if }\left(G_{n}(t)-L_{n}(t)-\Phi_{n}(t)\right)>0
\end{array}\right.
$$

(ii) Compute the DSM charging rate $P_{n}^{c}(t)$ of category $c$ with the lowest ratio

$$
\begin{aligned}
& \tilde{P}_{n}^{c}(t)=G_{n}(t)-L_{n}(t)-\Phi_{n}(t)-\tilde{S}_{n}^{c}(t) \\
& P_{n}^{c}(t)=\min \left\{P_{n}^{c,+}(t), \max \left\{P_{n}^{c,-}(t), \tilde{P}_{n}^{c}(t)\right\}\right\}
\end{aligned}
$$


$\tilde{S}_{n}^{c}=\sum_{c^{\prime}} P_{n}^{c^{\prime}}$ is the sum of charging rates of all categories with a lower ratio than $c$. For the category with the lowest ratio, it equals zero.

(iii) Compute the storage filling level of category $c$ for the next time step

$$
E_{n}^{c}(t+\Delta t)=\left\{\begin{array}{l}
\min \left\{E_{n}^{c}(t)+P_{n}^{c}(t) \Delta t, E_{n}^{c,+}(t+\Delta t)\right\}, \text { if } P_{n}^{c}(t) \geq 0 \\
\max \left\{E_{n}^{c}(t)+P_{n}^{c}(t) \Delta t, E_{n}^{c,-}(t+\Delta t)\right\}, \text { if } P_{n}^{c}(t)<0
\end{array}\right.
$$

(iv) Repeat steps (ii) and (iii) for the category with the next lowest ratio until the storage filling level for all categories was computed.

(v) Finally, compute the total interaction with the DSM storage at node $n, S_{n}(t)$, via

$$
S_{n}(t)=\sum_{n} P_{n}^{c}(t)
$$

Thus, charging and discharging rates are computed for the DSM categories in time dependent ascending order of the ratios of power capacity to remaining energy capacity. However, we believe that the choice of the distribution of charging among categories has little effect on the results because loads of all categories can only be shifted by up to one day. This section has summarised the most relevant details of the DSM approach from [34,40].

Figure 2 exemplarily shows the usage of the DSM in Germany for three days. Excess energy is stored in the DSM buffer, whereas deficits are covered from it. In the evening of the second day shown, the maximum of the energy capacity is reached. In the following hours, the energy capacity maximum is reduced, thereby forcing the DSM storage to discharge. Furthermore, the minimum charging rates are reached on day one and two.

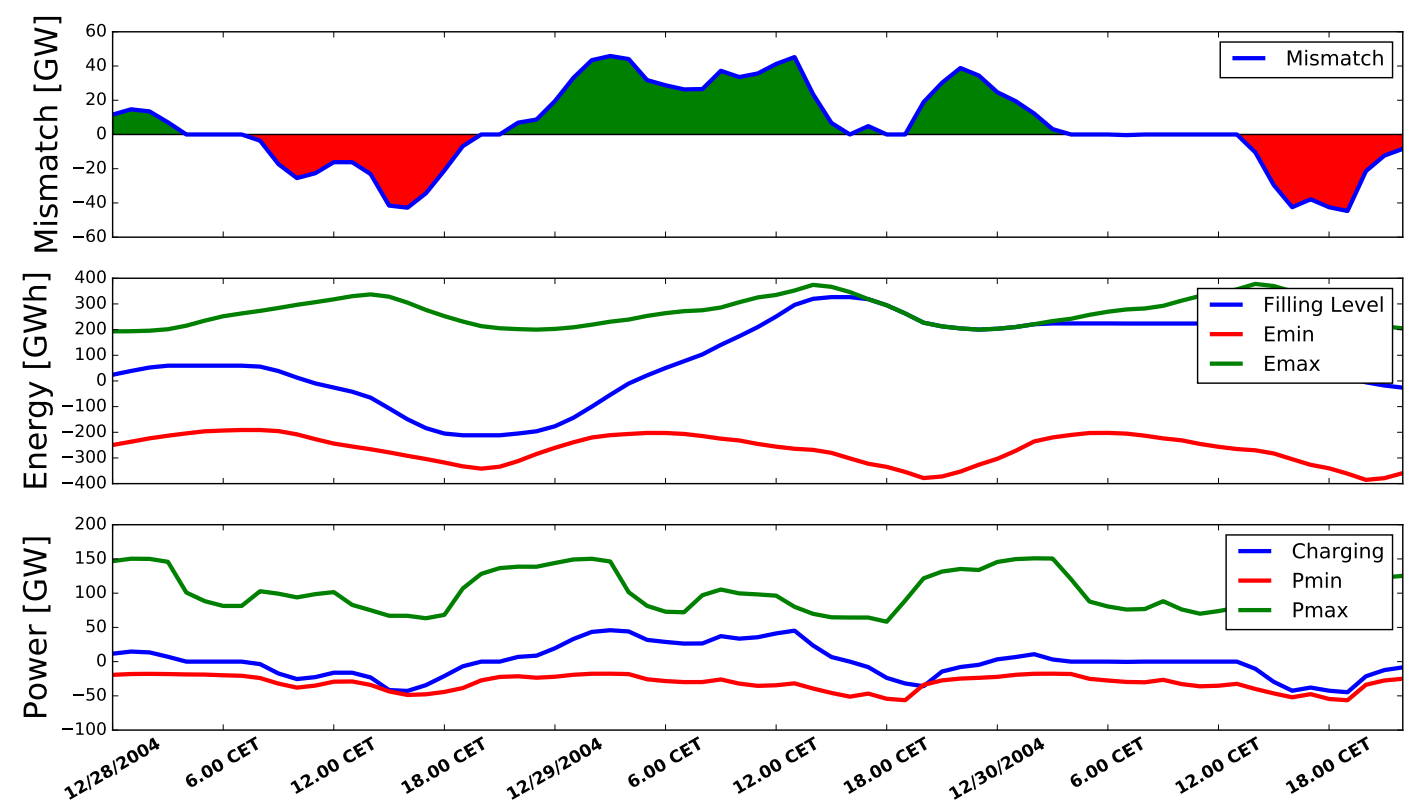

Figure 2. Mismatch (renewable generation-load-injection pattern), DSM filling level, and DSM charging rates for three exemplary days in Germany.

\subsection{Transmission}

Countries in our model are connected via inter-country transmission links. Hence, nodes can exchange excess energy and partially balance their mismatches. For transmission, the equations of a full electric power-flow in an alternating current (AC) electricity network are used in a common linear approximation [41] (occasionally referred to as DC approximation because the structure of the obtained equations is similar). Transmission is used prior to DSM, backup and curtailment and 
formulated as an optimisation problem consisting of two steps. The first step minimises the overall need for backup energy and the second step the dissipation by transmission. This can be interpreted as a cost-optimal dispatch strategy, if all nodes are assumed to have no limits on dispatchable generation and the same marginal cost, i.e., the same conventional generation technology is serving the load that cannot be supplied by renewable generation at any node in the system. The first step reads

$$
\begin{array}{ll}
\underset{\Phi(t)}{\operatorname{minimise}} & \sum_{n} B_{n}(t)=: B^{\min }(t) \\
\text { subject to } & \sum_{n} \Phi_{n}(t)=0 \\
& \mathrm{~F}_{l}^{-}<\left[K^{T} L^{+} \Phi(t)\right]_{l} \leq \mathrm{F}_{l}^{+}
\end{array}
$$

where $L^{+}$is the Moore-Penrose pseudo-inverse of the Laplacian, and $F_{l}^{ \pm}$are the limits imposed on the flow of link $l$ in both direction, which can, for example, be thermal limits.

The resulting need for backup energy is fixed for the second step. A second step is necessary because the solution $\Phi$ is generally not unique. This second step ensures the uniquety of the solution by minimising the dissipation of the flows ( $\propto F^{2}$ in a resistor network). It reads

$$
\begin{array}{ll}
\underset{\Phi(t)}{\operatorname{minimise}} & \sum_{l}\left[K^{T} L^{+} \Phi(t)\right]_{l}^{2} \\
\text { subject to } & \sum_{n} \Phi_{n}(t)=0 \\
& \mathrm{~F}_{l}^{-}<\left[K^{T} L^{+} \Phi(t)\right]_{l} \leq \mathrm{F}_{l}^{+} \\
& \sum_{n} B_{n}(t)=B^{\min }(t)
\end{array}
$$

The result is the injection pattern $\Phi(t)$ as the unique solution of the optimisation problem. The incidence matrix $K$ is defined as

$$
K_{n l}= \begin{cases}1 & \text { if link } 1 \text { begins at node } \mathrm{n} \\ -1 & \text { if link } 1 \text { ends at node } \mathrm{n} \\ 0 & \text { otherwise }\end{cases}
$$

and the Laplace Matrix $L$ is given by

$$
L_{n m}= \begin{cases}-1 & \text { if node } m \text { and } n \text { are connected by a link } \\ \operatorname{deg}\left(v_{n}\right) & \text { if } n=m \\ 0 & \text { otherwise }\end{cases}
$$

If the injection pattern $\Phi(t)$ is known, the flows can be computed via

$$
F=K^{T} L^{+} \Phi
$$

$K^{T} L^{+}$is often referred to as the PTDF (Power Transfer Distribution Factors) matrix. This transmission methodology is described in more detail in [42]. An equivalent formulation is used in [11,14,43-46].

\section{The Impact of DSM on Backup Energy Need}

We quantified the possible reduction of the need for backup energy in a fully renewable Europe $(\alpha=1.0)$ for two scenarios of transmission, which we refer to as vision 2030 (Vis.) and unlimited (Unl.), and two scenarios of capacity distribution, entitled homogeneous (Hom.) and inhomogeneous (Inh.). 
For unlimited transmission, no limits are imposed on transmission links (Equations (22) and (25)). Vision 2030 refers to the capacities as envisioned by ENTSO-E for 2030 [47]. Hence, these capacities are assigned as the imposed link constraints $F_{l}^{ \pm}$. In addition, symmetry is assumed: if $F_{l}^{+} \neq F_{l}^{-}$ in [47], both are set to $F_{l}^{ \pm}=\max \left\{F_{l}^{+}, F_{l}^{-}\right\}$.

The scenarios of capacity distribution differ by the distribution of the shares of renewables. In both cases, the mix of wind and PV generation capacities for each country is adopted from [48]. In the homogeneous scenario, however, each country covers its own load on average (i.e., $\alpha_{n}=1$ ), whereas in the inhomogeneous scenario, the ratio of generation capacities to load for each country are also taken from [48]. Shares of renewables from all countries are depicted in Figure 3. High shares of renewables can be observed in the inhomogeneous scenario for countries on the shores of the North Sea, such as Denmark or Great Britain.

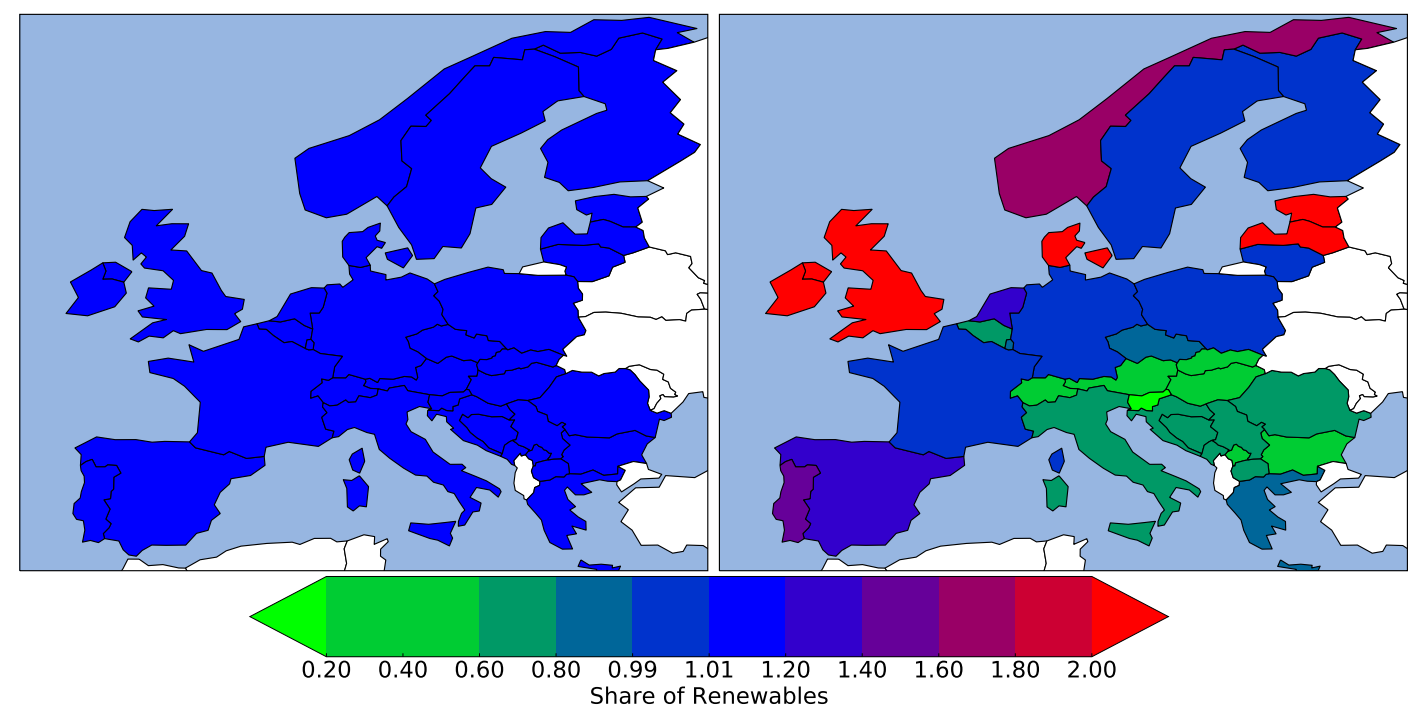

Figure 3. Shares of renewables ( $\alpha_{n}$, average renewable generation over consumption) for single countries in the homogeneous (left) and inhomogeneous (right) scenarios.

Figure 4 shows the need for backup energy and the possible reduction by DSM for all four scenarios. The complete bar shows the respective need for backup energy without DSM (e.g., ca. 16\% of the consumption in the case of homogeneous capacity distribution with unlimited transmission capacities). The blue component of each bar shows the corresponding reduction of the backup energy need by fully utilised DSM. If transmission is unlimited, backup energy need equals approximately $15 \%-16 \%$ of the total consumption in both scenarios. However, because DSM is not interacting with the inter-country transmission system, its potential is strongly reduced in the case of an inhomogeneous capacity distribution. In the inhomogeneous scenario, the need for backup energy can be reduced by only $15 \%$ compared to ca. one third in the homogeneous scenario because some countries produce more than they consume on average, and others produce less. Those with overproduction have a DSM buffer, which is full most of the time; those with little production relative to their consumption have a DSM buffer that is mostly empty. Thus, the uneven distribution of surpluses and deficits makes the usage of DSM less optimal in the inhomogeneous scenario.

If the transmission capacities are limited (vision 2030), two situations can be observed: first, the overall need for backup is increased by $45 \%$ in the homogeneous scenario and by $75 \%$ in the inhomogeneous. The reduction of the backup energy need grows as well (homogeneous: $+42 \%$, inhomogeneous: $+44 \%$ ), but not as much as the need for backup energy. Especially in the inhomogeneous scenario, transmission limitations hamper energy exchange to account for partial mismatches and thereby intensify the contrast between exporters and importers.

The frequency distribution of DSM energy filling levels (Figure 5) shows that DSM buffer is negative on average for all four scenarios. This is likely due to the higher number of hours with 
a negative mismatch ( $54 \%$ of hours for both capacity distributions). In addition, high levels of DSM storage filling of more than $1500 \mathrm{GWh}$ are never reached, if capacities are distributed inhomogeneously. This is caused by a large proportion of countries generating small amounts of renewable energy in this case, which is not sufficient to fill the buffer. On the lowest end of the scale, scenarios do not differ significantly. Below $-500 \mathrm{GWh}$, all scenarios show similar frequency distributions, which is likely due to the same occurrence of lasting periods without significant renewable generation. In these periods, neither the generation capacity distribution nor the transmission grid matters.

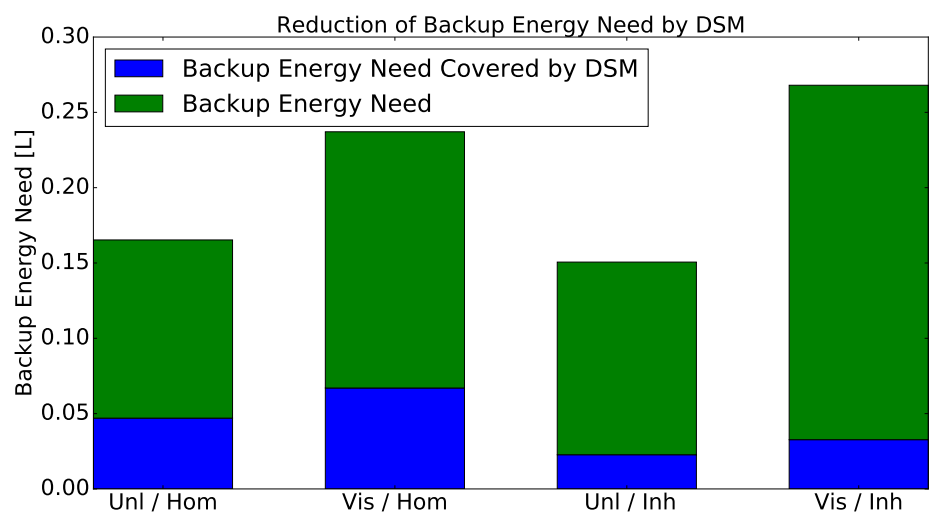

Figure 4. Need for backup energy and possible reduction by DSM. Entire bars show the need for backup energy without DSM. Blue components show the possible reduction by DSM. The backup energy need is measured in units of the overall consumption.

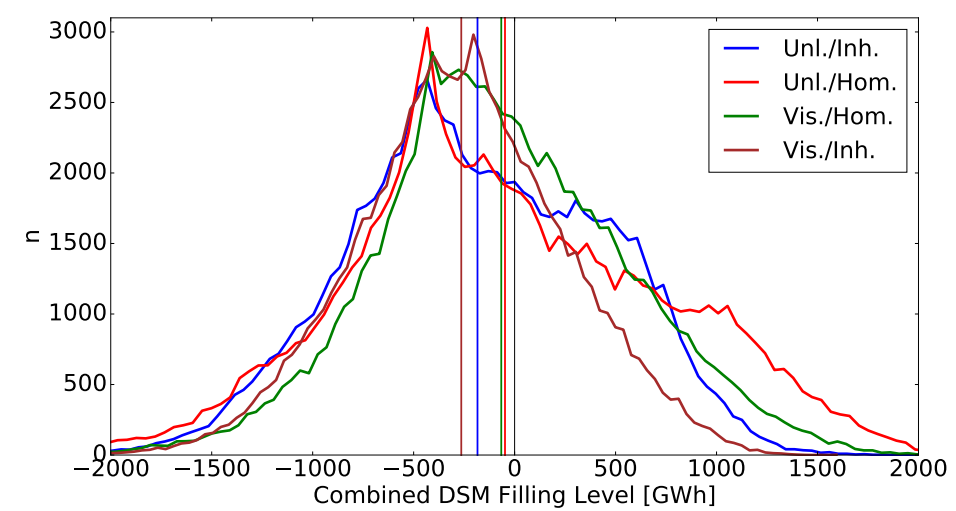

Figure 5. Frequency distribution of combined DSM storage filling levels. Vertical lines indicate the averages.

\section{Influence of DSM on the Optimal Mix of Wind and PV}

The optimal mix of different renewable sources in Europe has been investigated in different papers. In [3], the seasonal optimal mix is specified to be $40 \% \mathrm{PV}$ and $60 \%$ wind with respect to the monthly standard deviation, and, in [5], it is calculated at $20 \% \mathrm{PV}$ and $80 \%$ wind with respect to the backup energy need (note: we use the same generation and similar load data as [5]).

To calculate the optimal mix of wind and PV with and without DSM, we make major simplifications within this section: first, Europe is treated as a copper plate with homogeneous capacity distribution having only one time series of generation $G(t)=\sum_{n} G_{n}(t)$ and load $L(t)=\sum_{n} L_{n}(t)$. Second, DSM is treated like one large storage equivalent with time dependent constraints: 


$$
\begin{aligned}
& E^{+}(t)=\sum_{n, c} E_{n}^{c,+}(t) \\
& E^{-}(t)=\sum_{n, c} E_{n}^{c,-}(t) \\
& P^{+}(t)=\sum_{n, c} P_{n}^{c,+}(t) \\
& P^{-}(t)=\sum_{n, c} P_{n}^{c,-}(t)
\end{aligned}
$$

This simplification might violate the constraints imposed in Equations (10)-(13) and can therefore not be fully justified by assuming unlimited transmission between nodes and the possibility of exporting/importing energy to charge/discharge DSM storages at a different node. However, violations should rarely, if ever, occur, and the results can be interpreted as an upper limit of the potential benefit.

Figure 6 shows the need for backup energy with and without DSM. Without DSM, the optimal European mix with respect to the need for backup energy is $19 \% \mathrm{PV}$ and $81 \%$ wind. If DSM is fully utilised, this changes to an optimal mix of $36 \%$ PV and $64 \%$ wind. PV profits much more from DSM than wind. If the entire generation side is comprised of PV power, DSM can reduce the need for backup by ca. $40 \%$, whereas for a wind-only scenario, the possible reduction of the backup energy need is below $20 \%$. The reason for this is straightforward: Compared to wind, PV power has a deterministic diurnal cycle and therefore uses the DSM storage, which has properties that can be characterized as "daily storage" due to limited storage reservoir capacity more efficiently than wind. The more efficient usage of PV is also partly reflected by the seasonal variability of feed-in being the least if the solar generation share is higher than the wind share [49]. The red curve in Figure 6 shows the difference between the backup energy need with and without fully utilised DSM and thus the potential benefit from DSM. Three phases can be seen: first, it remains flat to a solar share of $20 \%$. Second, it increases steadily up to a solar share of ca. $60 \%$, and, third, it remains nearly unaltered up to a solar share of $100 \%$. Above a solar share of $60 \%$, the potential for DSM to reduce the need for backup energy is fully exploited.

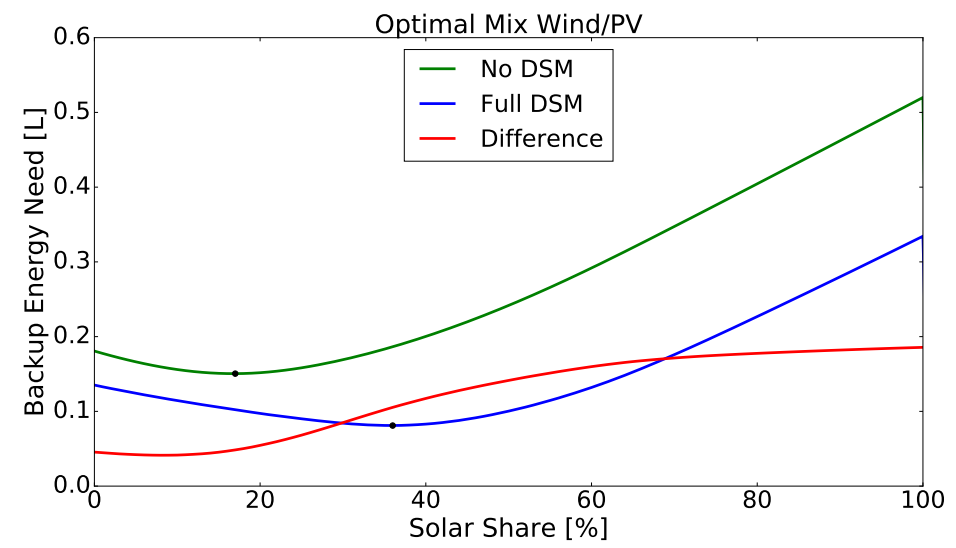

Figure 6. Backup energy need in dependency of the mix of wind and PV in Europe. Europe is treated like a copper plate. Black dots indicate the minima. The red line shows the difference between both curves. The backup energy need is measured in units of the overall consumption.

\section{DSM Potential vs. Share of Renewables}

The last investigated question of this paper is: until which share of renewables can DSM virtually cover the whole need for backup energy? We assume that the remaining share $1-\alpha$ is covered by a perfectly flexible dispatchable generation. This means that the dispatchable generation in our model has no must-run or ramping constraints. This question is important because the share of renewables 
in the European electricity mix has barely reached $25 \%$, but it continues growing and is expected to reach values of more than $80 \%$ by 2050 .

The need for backup energy in dependency of the share of renewables $\alpha$ is shown in Figure 7. Generation capacities are distributed homogeneously $\left(\alpha_{n}=\alpha \forall n\right)$ to reduce the need for backup energy among countries, and transmission is assumed to be unlimited between nodes. However, DSM is only used, like backup, locally after transmission and cannot be exchanged between countries. This means, for example, that after transmission, excess energy at node $a$ can not be transferred to node $b$ to charge the DSM buffer there. The inter-country transmission system is solely used to cover residual loads and does not interact with DSM. This can be justified because planned reinforcement measures of inter-country transmission links focus on the exchange of renewable energy and not on backup or storage energy. For up to a share of renewables of $67 \%$, all need for backup energy can be covered by DSM. Without DSM, the need for backup energy at a renewable share of $65 \%$ equals $2 \%$ of the yearly consumption (approximately $70 \mathrm{TWh}$ ).

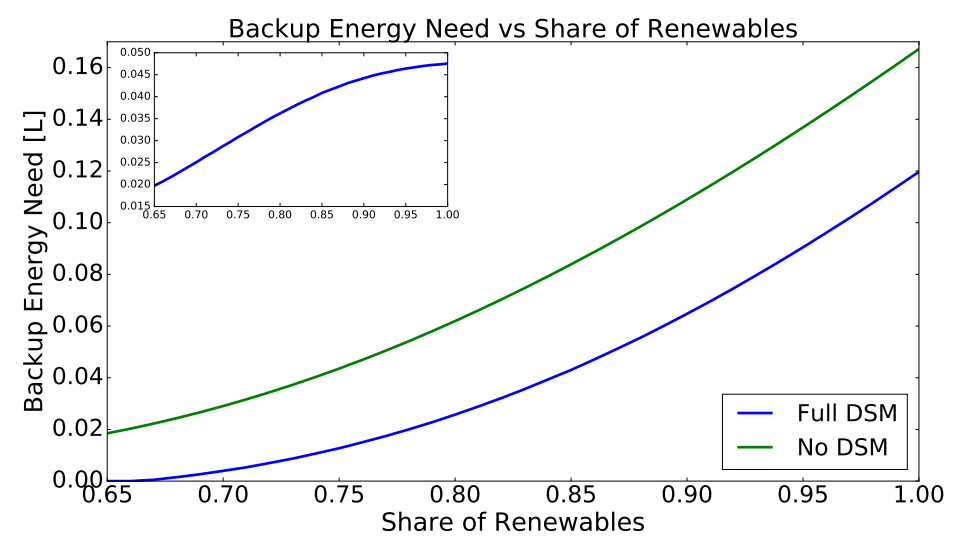

Figure 7. Need for backup energy in dependency of the renewable share in a European power system. Transmission capacities are assumed to be unlimited. The enclosed figure shows the difference between the two curves. The backup energy need is measured in units of the overall consumption.

Figure 7 (subfigure) also shows the reduction in backup energy need by DSM in units of the consumption. For $\alpha=0.65$, ca. $2 \%$ relative to the consumption could be replaced. This value continues to increase steadily over the interval $\alpha \in[0.65,1.0]$ and reaches its maximum at $4.8 \%$ for $\alpha=1$.

\section{Conclusions}

We have simulated a simplified highly renewable European power system to investigate the possible impact of demand side management on the need for backup energy. We have defined two scenarios of capacity distribution. In the homogeneous scenario, each country on average produces as much from renewable sources as it consumes, and, in the inhomogeneous scenario, installed capacities are distributed unevenly among European countries. First, it was shown that DSM can reduce the need for backup energy in a fully renewable European power system in dependency of the scenario by up to one third. Second, the optimal mix of wind and PV was found to be shifted from 19\% $\mathrm{PV}$, and $81 \%$ wind without DSM, to $36 \% \mathrm{PV}$ and $64 \%$ wind, if DSM is fully utilised. The beneficial interaction of wind and PV is also reflected by the fact that a wind-only Europe could reduce its backup need through the use of DSM by merely $20 \%$, whereas this number doubles in a PV-only scenario. Therefore, it can be concluded that the importance and economic performance of PV can be substantially increased, if the role of DSM within the energy market becomes vital.

It was also shown that DSM can theoretically cover all needs for backup energy up to a renewable share of ca. $67 \%$. This is still far from the current share of renewables in the European electricity mix of around $25 \%$. It is comparable to the reduction by a lossless storage in a fully renewable copper plate 
Europe with a storage size equal to three hours of the average load (ca. 1.2 TWh) [50]. This raises the question of what the interplay of DSM with its "daily storage" characteristics and European hydropower, with its large storage reservoir capacities and the seasonal dependency of its natural inflow, would look like. We believe that this is an interesting extension of this existing work.

DSM was shown to be an appropriate means of compensating the variable nature of renewable power sources such as wind and photovoltaics. Therefore, we conclude that demand side management has the potential to contribute significantly to issues that arise with the energy transition, which are currently in sight. This requires the implementation of a proper market environment and an appropriate understanding of risks and benefits by industry and policy makers.

Acknowledgments: The work is part of the RESTORE 2050 project (Wuppertal Institute, Next Energy, University of Oldenburg) that is financed by the Federal Ministry of Education and Research (BMBF, Fkz. 03SFF0439A). The work was also supported by the Ministry for Science and Culture of Lower Saxony (Hanover, Germany) in the 'ventus efficiens' project. We thank our project partners for helpful discussions and the provision of load and DSM data. Furthermore, we thank Martin Greiner (Aarhus) for helpful suggestions. We thank the editor and two anonymous reviewers for their constructive comments and suggestions, which helped us to improve the manuscript.

Author Contributions: All authors have discussed the findings, contributed to the writing of this article and approved the final manuscript.

Conflicts of Interest: The authors declare no conflict of interest.

\section{Abbreviations}

$n \in N \quad$ European country/node of the network

$G_{n}(t) \quad$ generation time series from renewable sources of wind and PV of country $n$

$L_{n}(t) \quad$ scheduled load time series of country $n$

$\Delta_{n}(t) \quad$ generation-load mismatch time series of country $n$

$\Phi_{n}(t) \quad$ injection pattern time series of country $n$

$B_{n}(t) \quad$ backup time series of country $n$

$C_{n}(t) \quad$ curtailment time series of country $n$

$S_{n}(t) \quad$ DSM charging/discharging time series of country $n$

$P_{n}^{c}(t) \quad$ DSM charging rate of country $n$ and load category $c$

$B_{n}^{E} \quad$ backup energy need of country $n$

$\alpha_{n} \quad$ share of renewables of country $n$

$R_{n}^{c}(t) \quad$ realised load time series of country $n$

$E_{n}^{c, \pm}(t) \quad$ time series of DSM storage energy limits

$P_{n}^{c, \pm}(t) \quad$ time series of DSM power limits

$E_{n}^{c}(t) \quad$ time series of DSM energy filling level

$K \quad$ incidence matrix of the network

$L \quad$ Laplace matrix of the network

$F_{l}^{ \pm} \quad$ link transmission constraints in both directions

\section{References}

1. Lund, H. Large-scale integration of wind power into different energy systems. Energy 2005, 30, 2402-2412.

2. Lund, H. Large-scale integration of optimal combinations of PV, wind and wave power into the electricity supply. Renew. Energy 2006, 31, 503-515.

3. Heide, D.; von Bremen, L.; Greiner, M.; Hoffmann, C.; Speckmann, M.; Bofinger, S. Seasonal optimal mix of wind and solar power in a future, highly renewable Europe. Renew. Energy 2010, 35, 2483-2489.

4. François, B.; Hingray, B.; Raynaud, D.; Borga, M.; Creutin, J. Increasing climate-related-energy penetration by integrating run-of-the river hydropower to wind/solar mix. Renew. Energy 2016, 87, 686-696.

5. Kies, A.; Nag, K.; von Bremen, L.; Lorenz, E.; Heinemann, D. Investigation of balancing effects in long term renewable energy feed-in with respect to the transmission grid. Adv. Sci. Res. 2015, 12, 91-95.

6. Santos-Alamillos, F.; Pozo-Vázquez, D.; Ruiz-Arias, J.; Von Bremen, L.; Tovar-Pescador, J. Combining wind farms with concentrating solar plants to provide stable renewable power. Renew. Energy 2015, 76, 539-550. 
7. Tafarte, P.; Das, S.; Eichhorn, M.; Thrän, D. Small adaptations, big impacts: Options for an optimized mix of variable renewable energy sources. Energy 2014, 72, 80-92.

8. Kies, A.; Schyska, B.U.; von Bremen, L. The optimal share of wave power in a highly renewable power system on the Iberian Peninsula. Energy Rep. 2016, 2, 221-228.

9. Weitemeyer, S.; Kleinhans, D.; Vogt, T.; Agert, C. Integration of renewable energy sources in future power systems: The role of storage. Renew. Energy 2015, 75, 14-20.

10. Weitemeyer, S.; Kleinhans, D.; Wienholt, L.; Vogt, T.; Agert, C. A European perspective: Potential of grid and storage for balancing renewable power systems. Energy Technol. 2015, 4, 114-122.

11. Heide, D.; Greiner, M.; von Bremen, L.; Hoffmann, C. Reduced storage and balancing needs in a fully renewable European power system with excess wind and solar power generation. Renew. Energy 2011, 36, 2515-2523.

12. Huber, M.; Dimkova, D.; Hamacher, T. Integration of wind and solar power in Europe: Assessment of flexibility requirements. Energy 2014, 69, 236-246.

13. Schlachtberger, D.; Becker, S.; Schramm, S.; Greiner, M. Backup flexibility classes in emerging large-scale renewable electricity systems. Energy Convers. Manag. 2016, 125, 336-346.

14. Becker, S.; Rodriguez, R.; Andresen, G.; Schramm, S.; Greiner, M. Transmission grid extensions during the build-up of a fully renewable Pan-European electricity supply. Energy 2014, 64, 404-418.

15. Brown, T. Transmission network loading in Europe with high shares of renewables. IET Renew. Power Gener. 2015, 9, 57-65.

16. Rabl, V.A.; Gellings, C.W. The concept of demand-side management. In Demand-Side Management and Electricity End-Use Efficiency; Springer: Heidelberg, Germany, 1988; pp. 99-112.

17. Strbac, G. Demand side management: Benefits and challenges. Energy Policy 2008, 36, 4419-4426.

18. Diamond, A.P. Energy Glossary; Nova Science Publishers: New York, NY, USA, 2002.

19. Atzeni, I.; Ordóñez, L.G.; Scutari, G.; Palomar, D.P.; Fonollosa, J.R. Demand-side management via distributed energy generation and storage optimization. IEEE Trans. Smart Grid 2013, 4, 866-876.

20. Stötzer, M.; Gronstedt, P.; Styczynski, Z. Demand side management potential-A case study for Germany. In Proceedings of the 21st International Conference on Electricity Distribution, Frankfurt, Germany, 6-9 June 2011.

21. Paulus, M.; Borggrefe, F. The potential of demand-side management in energy-intensive industries for electricity markets in Germany. Appl. Energy 2011, 88, 432-441.

22. Mesarić, P.; Krajcar, S. Home demand side management integrated with electric vehicles and renewable energy sources. Energy Build. 2015, 108, 1-9.

23. Emec, S.; Kuschke, M.; Chemnitz, M.; Strunz, K. Potential for demand side management in automotive manufacturing. In Proceedings of the 2013 4th IEEE/PES Innovative Smart Grid Technologies Europe (ISGT EUROPE), Lyngby, Denmark, 6-9 October 2013; IEEE: Piscataway, NJ, USA; pp. 1-5.

24. Klobasa, M. Analysis of demand response and wind integration in Germany's electricity market. IET Renew. Power Gener. 2010, 4, 55-63.

25. Lund, H.; Kempton, W. Integration of renewable energy into the transport and electricity sectors through V2G. Energy Policy 2008, 36, 3578-3587.

26. Stadler, I. Power grid balancing of energy systems with high renewable energy penetration by demand response. Util. Policy 2008, 16, 90-98.

27. Moura, P.S.; De Almeida, A.T. The role of demand-side management in the grid integration of wind power. Appl. Energy 2010, 87, 2581-2588.

28. Pollhammer, K.; Kupzog, F.; Gamauf, T.; Kremen, M. Modeling of demand side shifting potentials for smart power grids. In Proceedings of the 2011 AFRICON, Livingstone, Zambia, 13-15 September 2011; IEEE: Piscataway, NJ, USA, 2011; pp. 1-5.

29. Palensky, P.; Dietrich, D. Demand side management: Demand response, intelligent energy systems, and smart loads. IEEE Trans. Ind. Inform. 2011, 7, 381-388.

30. Göransson, L.; Goop, J.; Unger, T.; Odenberger, M.; Johnsson, F. Linkages between demand-side management and congestion in the European electricity transmission system. Energy 2014, 69, 860-872.

31. Zerrahn, A.; Schill, W.P. On the representation of demand-side management in power system models. Energy 2015, 84, 840-845. 
32. Tröster, E.; Kuwahata, R.; Ackermann, T. European Grid Study 2030/2050; Greenpeace International: Langen, Germany, 18 January 2011.

33. Kohler, S.; Agricola, A.; Seidl, H. Dena Grid Study IIe_Integration of Renewable Energy Sources in the German Power Supply System from 2015-2020 with an Outlook to 2025; Deutsche Energie-Agentur (dena), German Energy Agency: Berlin, Germany, 2010.

34. Kleinhans, D. Towards a systematic characterization of the potential of demand side management. 2014, arXiv:1401.4121.

35. Rienecker, M.M.; Suarez, M.J.; Gelaro, R.; Todling, R.; Bacmeister, J.; Liu, E.; Bosilovich, M.G.; Schubert, S.D.; Takacs, L.; Kim, G.K.; et al. MERRA: NASA's modern-era retrospective analysis for research and applications. J. Clim. 2011, 24, 3624-3648.

36. Cano, D.; Monget, J.; Albuisson, M.; Guillard, H.; Regas, N.; Wald, L. A method for the determination of the global solar radiation from meteorological satellite data. Sol. Energy 1986, 37, 31-39.

37. Hammer, A.; Heinemann, D.; Westerhellweg, A. Derivation of daylight and solar irradiance data from satellite observations. In Proceedings of the 9th Conference on Satellite Meteorology and Oceanography, Paris, France, 25-29 May 1998; pp. 747-750.

38. Klucher, T. Evaluation of models to predict insolation on tilted surfaces. Sol. Energy 1979, 23, 111-114.

39. Kies, A.; Chattopadhyay, K.; von Bremen, L.; Lorenz, E.; Heinemann, D. Simulation of renewable feed-in for power system studies; In RESTORE 2050 Project Report; Technical Report; NEXT ENERGYEWE-Forschungszentrum für Energietechnologie: Oldenburg, Germany, 2016.

40. Meyer, K.; Kleinhans, D. Arbeitspaket 5: Lastmanagement charakterisierung und quantifizierung des lastmanagementpotentials fuer Europa. In Restore 2050; Technical Report; NEXT ENERGYEWE-Forschungszentrum für Energietechnologie: Oldenburg, Germany, 2015.

41. Oeding, D.; Oswald, B.R. Elektrische Kraftwerke und Netze; Springer: Berlin/Heidelberg, Germany, 2004; Volume 6.

42. Heide, D. Statistical Physics of Power Flows on Networks with a High Share of Fluctuating Renewable Generation. Ph.D. Thesis, Johann Wolfgang Goethe-Universitaet Frankfurt am Main, Frankfurt, Germany, 2010.

43. Rodriguez, R.A.; Becker, S.; Greiner, M. Cost-optimal design of a simplified, highly renewable pan-European electricity system. Energy 2015, 83, 658-668.

44. Kies, A.; von Bremen, L.; Chattopadhyay, K.; Lorenz, E.; Heinemann, D. Backup, storage and transmission estimates of a supra-European electricity grid with high shares of renewables. In Proceedings of the 14th Wind Integration Workshop, Brussels, Belgium, 20-22 October 2015.

45. Rodriguez, R.A.; Becker, S.; Andresen, G.B.; Heide, D.; Greiner, M. Transmission needs across a fully renewable European power system. Renew. Energy 2014, 63, 467-476.

46. Kies, A.; Schyska, B.; von Bremen, L. Curtailment in a highly renewable power system and its effect on capacity factors. Energies 2016, 9, 510 .

47. European Network of Transmission System Operators for Electricity. Ten-Year Network Development Plan 2016; European Network of Transmission System Operators for Electricity: Brussels, Belgium, 2016.

48. Pfluger, B.; Sensfuß, F.; Schubert, G.; Leisentritt, J. Tangible Ways towards Climate Protection in the European Union (EU Long-Term Scenarios 2050); Fraunhofer Institut für System-und Innovationsforschung ISI: Karlsruhe, Germany, 2011.

49. Bett, P.E.; Thornton, H.E. The climatological relationships between wind and solar energy supply in Britain. Renew. Energy 2016, 87, 96-110.

50. Rasmussen, M.G.; Andresen, G.B.; Greiner, M. Storage and balancing synergies in a fully or highly renewable pan-European power system. Energy Policy 2012, 51, 642-651.

(C) 2016 by the authors; licensee MDPI, Basel, Switzerland. This article is an open access article distributed under the terms and conditions of the Creative Commons Attribution (CC-BY) license (http://creativecommons.org/licenses/by/4.0/). 